\title{
Reflection property of nano-acoustic wave at the air/GaN interface
}

\author{
Chia-Lung Hsieh, Kung-Hsuan Lin, and Shr-Bin Wu \\ Graduate Institute of Electro-Optical Engineering, National Taiwan University, Taipei 10617, Taiwan
}

Chang-Chi Pan and Jen-Inn Chyi

Optical Sciences Center, National Central University, Chung-Li 32054, Taiwan

Chi-Kuang Sun ${ }^{\text {a) }}$

Department of Electrical Engineering and Graduate Institute of Electro-Optical Engineering, National Taiwan University, Taipei 10617, Taiwan

(Received 29 March 2004; accepted 15 September 2004)

\begin{abstract}
We present a study on the total internal reflection of nano-acoustic waves (NAWs) at the air/GaN interface. The coherent NAW was generated and detected by piezoelectric InGaN/GaN multiple quantum wells using a femtosecond transient transmission technique. With suitably designed sample structures, the fact that strain of the NAW experiences a sign change after total internal reflection at the air/solid interface is examined directly. The surface roughness of the sample was found to distort the wave front of the NAW and to diminish the measured amplitude of the reflected NAW. (C) 2004 American Institute of Physics. [DOI: 10.1063/1.1819989]
\end{abstract}

Zone-folded coherent acoustic phonons generated in semiconductor with artificial periodic structures have been demonstrated by several groups. ${ }^{1-8}$ Recently, we reported that the amplitude of the measured coherent LA oscillation was enhanced by several orders due to the strong piezoelectric effect in GaN-based systems. ${ }^{4-8}$ Because the coherence of the zone-folded acoustic phonons in time is high, thermal and quantum fluctuations can be neglected. Therefore, it can be treated as a coherent acoustic wave with a nanometerscale wavelength and can be modeled by a macroscopic continuum elastic theory. ${ }^{9}$ Consequently, the piezoelectric semiconductor with periodic strained layers can be treated as an optical piezoelectric transducer to generate nano-acoustic waves (NAWs). Analogous to a typical electronic piezoelectric transducer that generates acoustic waves propagating in two counter directions, an optical femtosecond pulse also induces NAW propagating in two counter-directions. For a typical transducer, one side is the wave source output and the other side should be carefully designed to avoid the interference between the output signals and the reflection of waves propagating in the other direction. For example, a thick impedance-matching material with high attenuation is usually adopted to eliminate the reflected waves. Another way is to combine the two direction waves into one by an acoustic mirror. With an appropriate design, one can generate the acoustic wave propagating only in one direction.

In this letter, we treat periodic nano-strained-layer structure in a piezoelectric semiconductor as an optical NAW transducer with an acoustic mirror placed at one side, accomplished through the total internal reflection at the air/sample interface. With a suitably designed sample structure, the phase property of the reflected NAW on air/solid interface is examined. Our study not only provides the design guideline for future nano piezoelectric transducers, but also reveals the fact that strain of the NAW experiences a $180^{\circ}$ sign change after total internal reflection at the air/solid interface. The surface roughness of the sample, on the order of several nanometers, was found to distort the wave front of the NAW

\footnotetext{
${ }^{a}$ Author to whom correspondence should be addressed; electronic mail: sun@cc.ee.ntu.edu.tw
}

and to significantly diminish the measured NAW amplitude.

In our study, $\operatorname{In}_{0.23} \mathrm{Ga}_{0.77} \mathrm{~N} / \mathrm{GaN}$ multiple quantum well (MQW) was the periodic nano-strained-layer structure (see Fig. 1). We have grown two MQW samples, both consisted of ten periods of InGaN/GaN QWs on a 3.4- $\mu$ m-thick undoped GaN buffer layer and a $c$-plane sapphire substrate, ${ }^{10}$ for this specific study. Sample A consists of ten periods of $2.9 \mathrm{~nm} / 21.7 \mathrm{~nm} \mathrm{In}{ }_{0.23} \mathrm{Ga}_{0.77} \mathrm{~N} / \mathrm{GaN}$ QWs and is capped with a $4.7 \mathrm{~nm}$ GaN layer (average value). Since the acoustic wavelength is determined by the period of the MQW, the

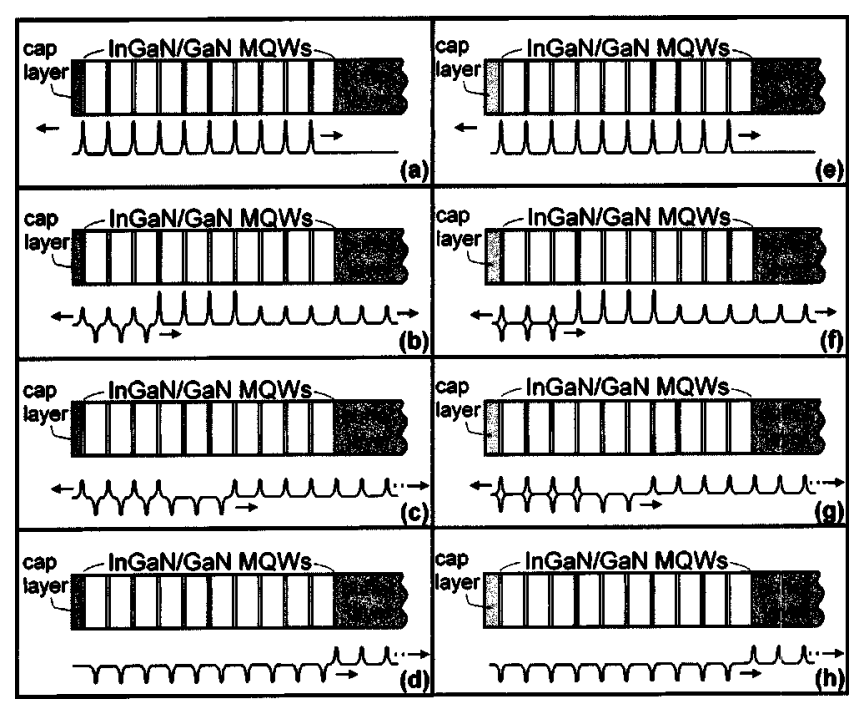

FIG. 1. Time-sequenced illustrations showing NAW propagating in a InGaN/GaN MQW after the generation of the NAW by an optical femtosecond pulse at time zero. With appropriate cap-layer design, two counterpropagating waves can be connected in one direction inphase, as shown in (a)-(d) [corresponding to (1/4) $\lambda$ cap-layer thickness] or out of phase as shown in (e)-(h) [corresponding to (1/2) $\lambda$ cap-layer thickness]. (a) At time zero, strain of the NAW is initiated in wells and propagating in two counterdirections. (b) At three periods of time after time zero, the wave propagating toward substrate is leaving the MQW region, and part of the wave propagating toward the acoustic mirror is totally internally reflected and follows the other wave propagating toward the substrate. (c) The NAW propagation condition at six periods of time after time zero. (d) The NAW propagation condition at ten periods of time after time zero. (e)-(h) are analogous to (a)-(d), but with different cap-layer thickness. 
total distance from the center of the first well to the acoustic mirror is designed to be $6.15 \mathrm{~nm}$, corresponding to onequarter of the acoustic wavelength. Sample B consists of ten periods $2.9 \mathrm{~nm} / 13.0 \mathrm{~nm} \operatorname{In}_{0.23} \mathrm{Ga}_{0.77} \mathrm{~N} / \mathrm{GaN}$ QWs and is capped with a 7.9-nm-thick (average value) GaN layer. In this case the total distance from the center of the first well to the air interface (acoustic mirror) is $9.35 \mathrm{~nm}$, corresponding to $\sim 0.59$ wavelength.

When the strained MQW were excited by an optical femtosecond pulse, photoexcited carriers will initiate two strain waves propagating in counter-directions through piezoelectric and deformation couplings. ${ }^{9}$ Since the terahertzto-subterahertz acoustic modes do not exist in air, the air/ solid interface is a perfect acoustic mirror to totally reflect the NAW. Therefore, the thickness of the adjustment cap layer on the MQW structure determines the position of the acoustic mirror. The wave propagating toward the acoustic mirror will be reflected and follow the other one propagating toward the substrate. Due to the fact that the air/solid interface is a free-end interface, so the sign of strain waves is inversed (phase changes $180^{\circ}$ ) after reflection. On the other hand, the spatial displacement of the NAW experiences no phase change after being reflected at the free-end interface. With a suitable design of the cap-layer thickness, two counter-direction-propagating waves can be connected in one direction in phase or out of phase. For example, Figs. 1(a)-1(d) and Figs 1(e)-1(h) show two sets of timesequenced diagrams showing propagation of the NAW in the designed InGaN/GaN MQW samples for two cases with different cap-layer thicknesses. In the case shown in Figs. 1(a)-1(d), the thickness of the cap layer is designed to be one-quarter of the acoustic wavelength, so that after the surface reflection the two counter-direction-propagating waves would be connected in one direction with their strain in phase. In the other case shown in Figs. 1(e)-1(h), the thickness of cap layer is designed to be one-half of the acoustic wavelength, so that after the surface reflection, the two counter-direction-propagating waves would be connected in one direction with their strain out of phase.

We monitored propagation of the NAW in the piezoelectric transducer by measuring its transient optical transmission changes, ${ }^{5}$ since the strain-induced piezoelectric field will modulate the optical absorption of the InGaN MQW through the quantum-confined Franz-Keldysh effect. ${ }^{4}$ From this point of view, the MQW can be treated not only as a NAW generator but also as a detector. The optical transmission modulation due to strain of the NAW can be modeled as ${ }^{11}$

$$
\left(\frac{\Delta T(t)}{T}\right)_{\mathrm{NAW}}=\int_{-\infty}^{\infty} d z S(z, t) \times F(z, \omega),
$$

where $S(z, t)$ is the strain wave function and $F(z ; \omega)$ is the sensitivity function. According to Eq. (1), the optical transmission modulation caused by the NAW can be interpreted as an integral of a strain wave function $S(z, t)$ weighted by a sensitivity function $F(z ; \omega)$ (determined by the MQW structure) over the $z$ direction. Due to the selection of the probe wavelength, the value of the sensitivity function in our case is nonzero only in the wells, therefore, the optical transmission modulation due to NAW is revealed when the strain waves enter or leave the wells.

In the case of sample A, since the optical detection mechanism is sensitive to strain, it can be expected that the Downloaded 16 Feb 2009 to 140.112 .113 .225 . Redistribution subje
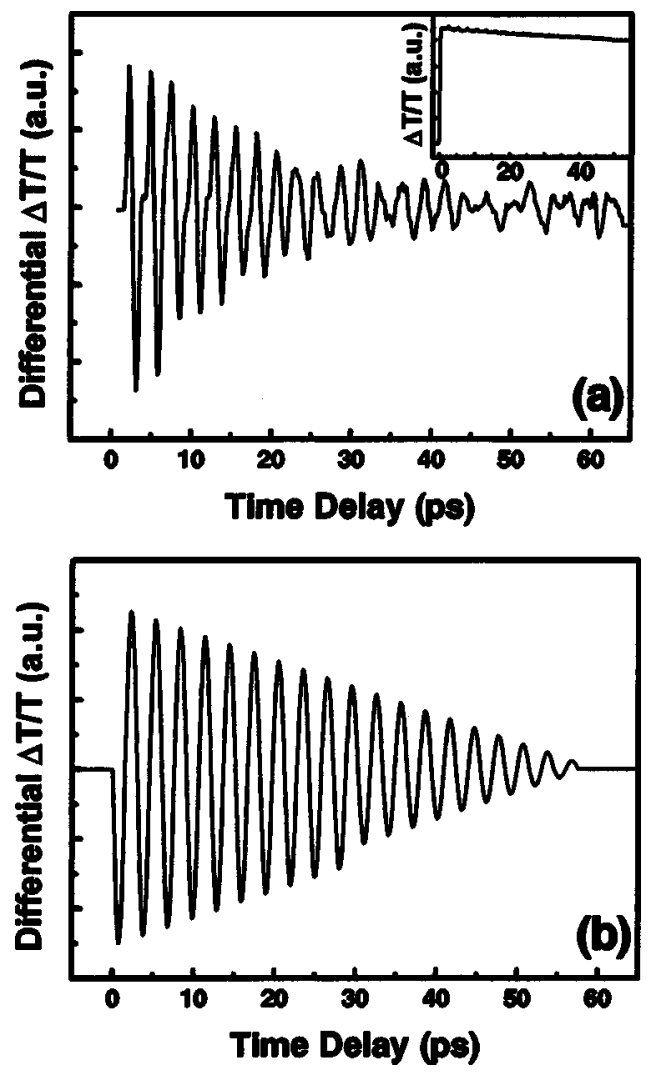

FIG. 2. (a) Differentiated trace of the measured optical transient transmission of sample A, showing a prolonged coherent oscillation. (b) Corresponding theoretical simulation.

reflected NAW will follow another NAW in phase due to a $180^{\circ}$ phase change and a total half-wavelength roundtrip. If the optical detection mechanism was sensitive to spatial displacement, there would be no phase change after the reflection and thus the reflected NAW would be expected to follow the other NAW $180^{\circ}$ out of phase. We recorded the transient optical transmission changes of sample A by a degenerate pump-probe system. ${ }^{5}$ The central wavelength of the optical femtosecond pulses was $390 \mathrm{~nm}$. The transmission change $\Delta T / T$ of sample A as a function of time delay is shown in Fig. 2(a). A 20-period in-phase oscillation is observed for sample A consisting of 10-period MQW. The doubled number of oscillation cycles is due to the fact that the reflected NAW follows the other NAW in phase.

The theoretical calculation of the differential transmission change as a function of time delay of sample $\mathrm{A}$ is shown in Fig. 2(b). In the calculation, we modeled the strain wave function $S(z, t)$ as positive parts of a sinusoidal function with ten periods. For simplicity but without losing capacity of examining physical mechanism, the sensitivity function $F(z ; \omega)$ is assumed to be a zero-one square wave function, following the MQW structure. As time evolves, the initial strain wave function $S(z, t)$ separates into two counterpropagating strain waves with equal amplitude. We assume the reflection at the air/sample interface is a perfect total internal reflection. The sound velocity used in simulation is 8100 $\mathrm{m} / \mathrm{s},{ }^{12}$ From Fig. 2(b), there are 20 peaks with a linearly decaying envelope. The theoretical calculation agrees quite well with experimental data, indicating the $180^{\circ}$ phase change after total internal reflection and the correct deposition of the cap-layer thickness $(1 / 4 \lambda)$ that produce an into AIP license or copyright; see http://apl.aip.org/apl/copyright.jsp 

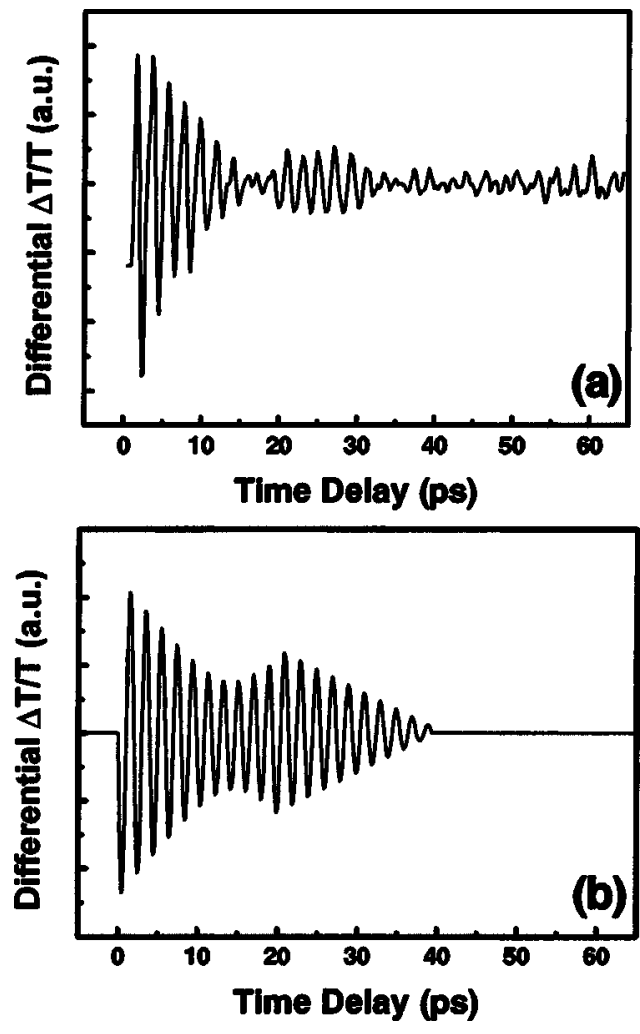

FIG. 3. (a) Differentiated trace of the measured optical transient transmission of sample B, showing a phase change of $240^{\circ}$. (b) Corresponding theoretical simulation.

phase prolonged NAW. From a different point of view, our study once again supports the fact that optical transmission intensity is sensitive to strain rather than spatial displacement.

In the case of sample B, the distance from the center of the first well to the air interface (acoustic mirror) corresponds to $\sim 0.59$ wavelength. The reflected NAW is thus expected to follow the initiated NAW with a $\sim 245^{\circ}$ phase difference. The measured differential transient transmission change in sample B is shown in Fig. 3(a). The amplitude of the oscillation first decays rapidly and grows again with a gradual $240^{\circ}$ phase change, showing a minimal amplitude of oscillations at 16.5 ps time delay, and then fades away. Since the optical transmission modulation reflects the total strain amplitude in ten quantum wells the out-of-phase refleccted NAW would cancel out the optical signal contributed from the initial NAW. As time evolves, the initial NAW propagating toward the substrate will leave the MQW region after ten oscillation cycles, and it can be expected that the strain of reflected out-of-phase NAW will dominate the observed optical signals. The amplitude of oscillations fades away when the reflected NAW also leaves the MQW region [as depicted in Figs. 1(e)-1(h)]. The observed $240^{\circ}$ phase change also agrees quite well with the expected value $\left(245^{\circ}\right)$.

The theoretical calculation of the differential transmission changes as a function of time delay of sample $B$ is shown in Fig. 3(b). In Fig. 3(b), we observed the minimal amplitude of oscillation at $\sim 14$ ps time delay with a $245^{\circ}$ gradual phase change after the minimum, similar to the experimental result. However, the minimal amplitude of oscillation from the experiment occurs at a later time (16.5 ps), indicating that the effective optical oscillation amplitude caused by the reflected acoustic wave seems to be attenuated. Downloaded 16 Feb 2009 to 140.112 .113 .225 . Redistribution subject
One may also notice that the experimental data of sample A in Fig. 2(a) decay more rapidly than theoretical calculation in Fig. 2(b), indicating the same fact. There are several possible reasons for the decrease of the optical signal. One reason is that the amplitude of reflective NAW is actually smaller than that of the initiated NAW (not 100\% reflection). Another reason is the distortion of the reflected acoustic wave front due to the sample surface roughness. Other factors include the propagation and scattering loss. Due to the great difference between the acoustic impedances of solid and air, unity reflectivity can be guaranteed. The acoustic lifetime and scattering effect, on the other hand, should play an equal role in two counterpropagating waves. In order to investigate the surface roughness effect, atomic force microscopy (Angscope SPM-A-100) measurement on the sample surface roughness was conducted and the rms values of surface roughness were found to be on the order of $0.34-1.93 \mathrm{~nm}$ and $0.77-2.63 \mathrm{~nm}$ for sample A and sample B, respectively. For an acoustic wave with a wavelength less than $25 \mathrm{~nm}$, this surface roughness condition can distort the reflected acoustic wave front. The energy of the strain pulses is thus dispersed in time and the measured optical signal amplitude will decrease. Another interesting issue one may note is that some residue oscillations seem to exist within the noise level after the reflected acoustic wave front leaves MQW region. These oscillations may be attributed to the zone-folding effect in the MQW.

In conclusion, with suitably designed sample structures, the phase property of the reflected NAW on air/solid interface is examined. Our study not only provides the design guideline for future nanopiezoelectric transducers, but also reveals the fact that strain of the NAW experiences a $180^{\circ}$ sign change after total internal reflection at the air/solid interface. The surface roughness of the sample was found to distort the wave front of NAW and to diminish the measured optical amplitude of reflected NAW.

The authors thank AngsNanoTek Taiwan Corp. for providing technical supports. This work was sponsored by National Science Council of Taiwan, under Grant NSC-932120-M-002-004, NSC-92-2112-M-002-044, and NSC-922215-E-008-020. One of the authors (K.-H.L.) would like to thank SiS Education Foundation for financial support.

${ }^{1}$ C. Colvard, T. A. Gant, M. V. Klein, R. Merlin, R. Fischer, H. Morkoc, and A. C. Gossard, Phys. Rev. B 31, 2080 (1985).

${ }^{2}$ A. Bartels, T. Dekorsy, H. Kurz, and K. Köhler, Phys. Rev. Lett. 82, 1044 (1999).

${ }^{3}$ K. Mizoguchi, M. Hase, S. Nakashima, and M. Nakayama, Phys. Rev. B 60, 8262 (1999)

${ }^{4}$ C.-K. Sun, J.-C. Liang, and X.-Y. Yu, Phys. Rev. Lett. 84, 179 (2000).

${ }^{5}$ C.-K. Sun, Y.-K. Huang, J.-C. Liang, A. Abare, and S. P. DenBaars, Appl. Phys. Lett. 78, 1201 (2001).

${ }^{6}$ J. S. Yahng, Y. D. Jho, K. J. Yee, E. Oh, J. C. Woo, D. S. Kim, G. D. Sanders, and C. J. Stanton, Appl. Phys. Lett. 80, 4723 (2002).

${ }^{7}$ G.-W. Chern, K.-H. Lin, Y.-K. Huang, and C.-K. Sun, Phys. Rev. B 67, 121303(R) (2003).

${ }^{8}$ C.-K. Sun, G.-W. Chern, K.-H. Lin, and Y.-K. Huang, Chin. J. Phys. (Taipei) 41, 643 (2003).

${ }^{9}$ G.-W. Chern, C.-K. Sun, G. D. Sanders, and C. J. Stanton, in Topics in Applied Physics: Ultrafast Dynamical Processes in Semiconductors, edited by K.-T. Tsen (Springer, Berlin, 2004), Vol. 92, pp. 339-390.

${ }^{10}$ C.-M. Lee, C.-C. Chuo, I.-L. Chen, J.-C. Chang, and J.-I. Chyi, IEEE Electron Device Lett. 24, 156 (2003).

${ }^{11}$ G.-W. Chern, K.-H. Lin, and C.-K. Sun, J. Appl. Phys. 95, 1114 (2004).

${ }^{12}$ H. Morkoc, Nitride Semiconductors and Devices (Springer, Berlin, 1999). to AIP license or copyright; see http://apl.aip.org/apl/copyright.jsp 